\title{
SEARCHING EVIDENCES FOR SPIRAL SHOCKS IN THE QUIESCENT ACCRETION DISK OF U GEM
}

\author{
V. V. Nonstruev, ${ }^{1}$ V. Chavushyan, ${ }^{2}$ and J. R. Valdés ${ }^{2}$ \\ RESUMEN
}

Encontranos que el disco de acreción en la etapa de quictud de U Gem, tiene una estructura complicada. A lo largo de la mancha brillante originada en la región de interacción entre el chorro y las partículas del disco, hay tambićn indicaciones de chodues espirales. El mapa Doppler y las variaciones en las separaciones del pico de las líncas de cmisión son indicativos de ello.

\section{ABSTRAC'T}

We find that the quiescent accretion disk of $U$ Gem has a complicated structure. Along to the bright spot originating in the region of interaction between the stream and the disk particles, there are also explicit inclications of spiral shocks. The Doppler map and the variations of the peak separation of the emission lines are inclicative.

\section{liry Wotds: ACCRETION, ACCRETION DISKS - LINE: PROFILES - NOVAE, CATACLYSMIC VARIABLES - SHOCK WAVES - STARS: INDIVIDUAL (U GEM)}

Although inclications for spiral shocks in the hot aceretion disks during an outburst have already been found (Sterghs et al. 1997), the problem on the spiral structure of the culieserent accretion disks still remains unsolved. The observational detection of spiral shocks in such disks would be very important since spiral arms are very efficient in transporting angular momentum into the outer part of the disk (Boffin 2001). However if existing, spiral shocks would be much more difficult to defect than the strong shocks in the hot aceretion disk during outburst. Veverthelesis. first steps towards an observational confirmation have been started. Neustroev \& Borisov (1998) and Neustroev ot al. (20)2) have found some evidences for spiral structure of the quiescent aceretion disk of U Gem and IP Peg. Further investigat ions in this area are strongly required.

Here we present new evidences for spiral shocks in the quiescent accretion disk of U Gem. High signal-to-noise and medium resolution $(\sim 3 \AA)$ opti(al spectra of 1 Gem were obtained on the 2.12-m trescope of the Observatorio Astrofisice "Guillermo Horo" (AOGH). ('anancal. México, during 2000 Norember 17, with a total coverage of 4.3 hours. A total of 24 spectria were taken in the warchength range 3900-5400 A with exposures of $600 \mathrm{~s}$. covering one orbital period.

The distribution of the acretion disk's cmission

\footnotetext{
1 Vational Eniversity of Ireland. Calway. Ireland.

${ }^{2}$ Instituto Nacional de Astrofísica Optica y Flectrónica, Puebla, Pue., México.
}

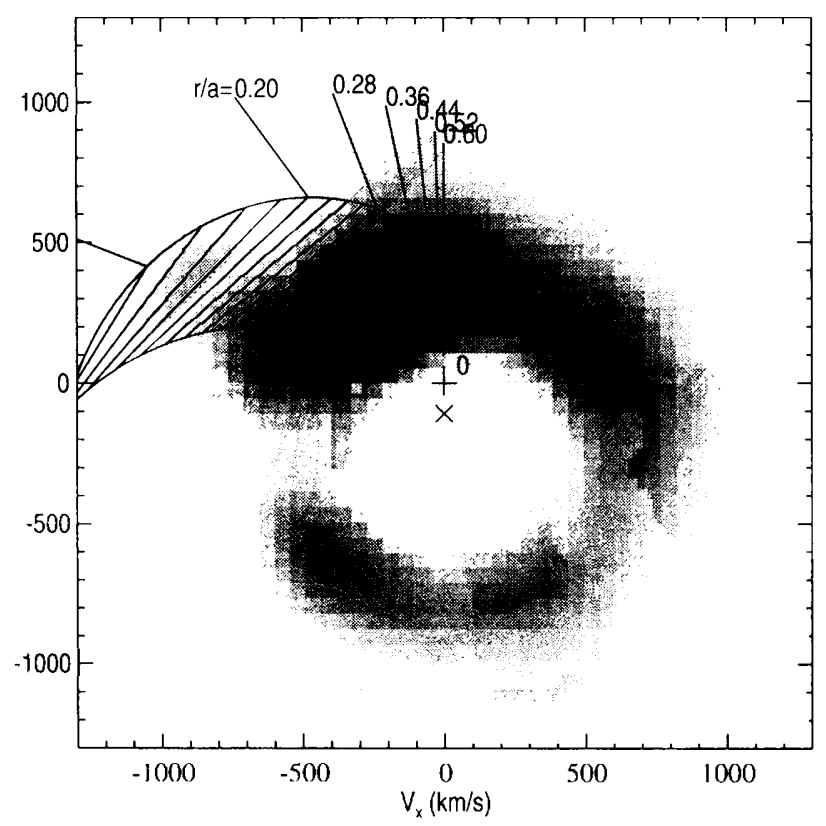

Fig. 1. The Doppler map of the $\mathrm{H} \beta$ emission from $U$ Gem in quiescence.

was explored by computing a Doppler map, using the method of Doppler tomography (Marsh \& Horne 1988). The H.3 tomogram shows the bright emitting region superposed on the typical ring-shaped emission of the accretion disk. This bright region can be unequivocally contributed to emission from the bright spot on the outer edge of the accretion disk. Note that the emission from the secondary is completely absent.

Disk emission is centered on the white dwarf and has small, but well noticeable azimuthal asymmetry 


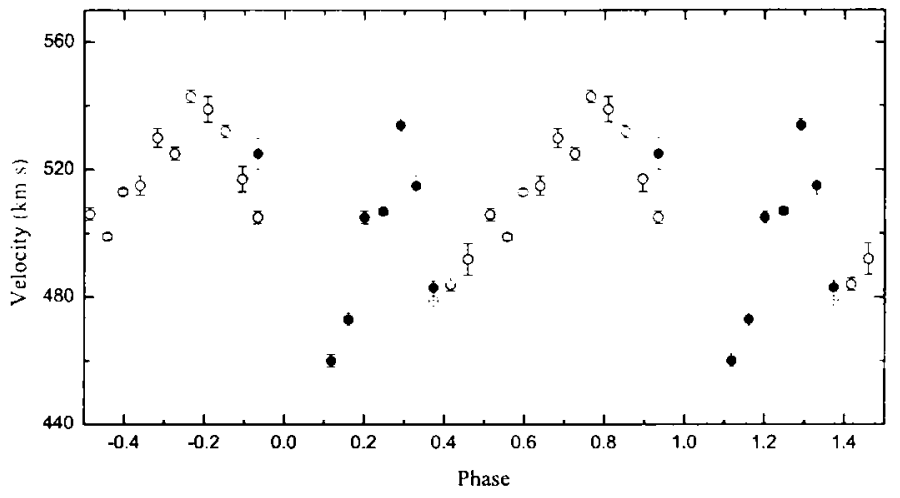

Fig. 2. The modulation of the duuble peak separation of the $\mathrm{H} \beta$ emission across the binary period. Filled and open circles correspond to the velocity difference betworn the center of the line and the blue and red peak respectively. These velocity differences were determined during orbital phases when the emission component from the bright spot was on the opposite peak of the line.

in the form of a two armed pattern. The line flux in the arms is about a factor of $\sim 1.4$ stronger than that of the disk emission outside these areas, pointing to some heating and density enhancement. Note that the marked areas of increased luminosity are not perfectly symmetric. The arm in the upper right of the tomogram is slightly stronger.

These arms are located too far from the region of interaction between the strean and the disk particles. None of the theories predict the presence of any bright spots hore. which are connected with such an interaction. Howerer, exactly in these areas of the Doppler maps there should be spiral shocks predicted numerically by a number of researchers (Sawada et al. 1986). Furthermore, very similar twoarmed structure was detected by Groot (2001) in the accretion disk of U Gem and by Steeghs et al. (1997) in IP Peg, when both systems were in outburst.

However, unlike Stecghs et al. (1997) and Groot (2001), we cannot confidently assert that the form of both arms is spiral. The reason of it can be the fact, that spiral shocks in quiescence should be tightly wrapped (Steeghs \& Stehle 1999). Henc' ( the areas on the tomograms corresponding to the spirals will little differ from a ring. In addition this difference will be difficult for detecting, taking into account low brightness of these shocks.

As additional observational evidence for spiral shocks in the accretion disk can be regarderl the modulation of the double peak separation of the emission lines across the binary orbit in a particular way (Neustroev \& Borisov 1998). The detertion of such modulation is complicated by presence of the s-wave component distorting the lines. To remove this influence, the sperctra of $L G$ ism were corrected

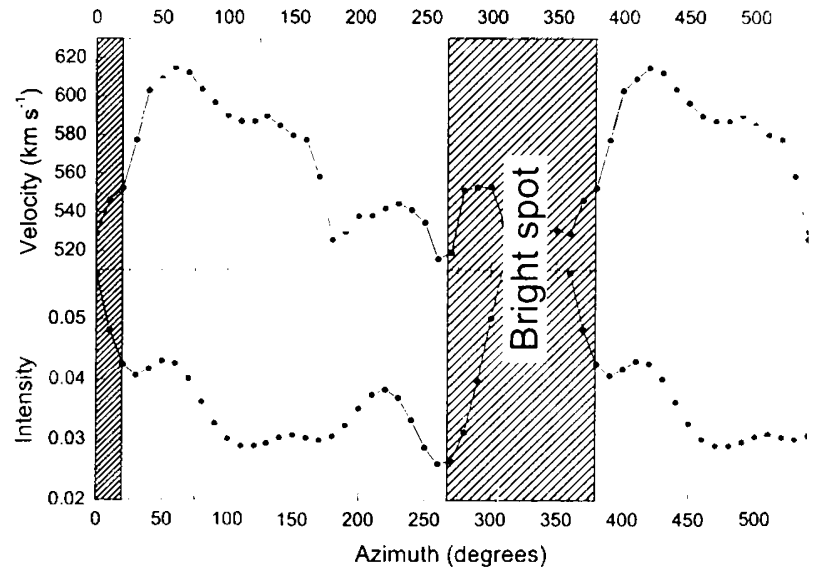

Fig. 3. Vartation in the velocity and omission strength of the spiral arms as a lunction of azimuth.

for wavelength shifts due to orbital motion. Ifter that we have determined, using galussian fitting. the radial indorities of only such line peaks that atre not garbled by the s-wave compencut Wo hare taken the absolute values of these velocities as the half of the double peak sepralations, and in Fig. 2 we shew their dependence on the orbital phase. One cant see that the double peak separation varies during the orbital period, at a firsi approximation as $\sin 2 \varphi$. These variations are the signature of $: \mathrm{m}=$-2 mocte in the accretion disk of $T^{+}$Gem. This mode can be recited by the tidal forcing and the deterter variations can be explained by the presence of spiral shorks in the disk, confirming the results of Nomstren a Burisos (1998). Additionally, we have also determined the variation in the velocity and emission strength of the (spiral) arms as a function of azinuth (Fig. 3). The obtained plot is qualitatively similar to fig. 4 that has been presented by Harlaft in of al. (1999) for spiral shocks in the arcretion disk of IP Pog during outburst.

Thus. we find that the quieserent accretion disk of U Gem has a complicated structure. Alomg to the bright spot originating in the rection of interaction between the stream and the disk particles. there are also explicit indications of spiral shock wares.

\section{REFEIRENC'FS}

Boffin, H.M.J., 2001, Lecture Noter in Physice 57:3. 69 Groot, P.J., 2001, Ap.J. 5.51, L89

Harlaftis, E.T. ret al., 19999. MNR.1S 306. 3ts

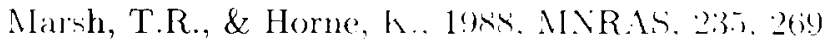

Veustroev, V.V., \& Borison, N.V., 199\%, .4.1. 336. L73

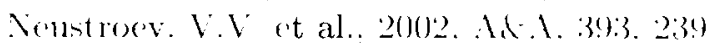

Sawada. K.. Matsuda. T., \& Hachisu, I., 1950, MNR. S 219.75

Stieglas. D., Harlaft is. F.T. \& Horne. K. 1999 MNRAS 290, L2X

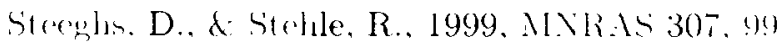

